\title{
Microwave Synthesis and Characterisation of Some Cycl [3, 2, 2] Azine Derivatives
}

\author{
M. Sheela Gopal ${ }^{1}$, I. Anitha ${ }^{2} *$ \\ ${ }^{1}$ TMJM Govt. College, Manimalakunnu, Ernakulam, Kerala- 686662, India \\ ${ }^{*}$ Postgraduate and Research Department of Chemistry, Maharaja's College, Ernakulam, Kerala- 682011, India \\ Tel.8089370720 Fax: 04842363038 \\ Email: dranithagirishkumar@gmail.com
}

\begin{abstract}
The synthesis and characterisation of few cycl[3.2.2]azine systems are described. They were obtained in moderate yields by microwave technique from 2-picoline, dimethyl acetylenedicarboxylate and phenacyl bromides in presence of a base. Antibacterial activity and fluorescence behaviour of these compounds are also described.
\end{abstract}

Keywords: Cycl[3, 2, 2]azine, Green chemistry, Antibacterial activity, Fluorescence

\section{Introduction}

The indolizine ring system found in natural products is an important structural skeleton in pharmaceutics because of their interesting biological properties [1]-[3]. Synthetic indolizines have found wide spread applications in drug designing, biological and pharmaceutical research fields [4][7]. Several reports are available showing the potential of indolizine derivatives as antimicrobial agent [8]. The cycl[3.2.2.]azine systems are tricyclic aromatic heterocycles with nitrogen as the central atom common to three rings and they are widely accepted due to their interesting physical and chemical properties[9]-[10]. Among cyclazine derivatives, tricyclic fused imidazo[1, 2-a]pyridines bridged between the $\mathrm{C}(3)$ and $\mathrm{C}(5)$ positions would be of particular interest because an imidazo[1, 2-a]pyridine ring system has popularly been utilized as a pharrmacophore for therapeutic drugs[11][13] and as biochemical fluorescent marker[14] due to its strong fluorescence[15]. The important characteristic feature of the cycl[3.2.2] azine is in the fact that the central nitrogen atom is nonbasic, indicating its pi-electrons are completely involved in the aromatic pi-electron system.

The synthesis of cyclazine derivatives has been actively investigated and many synthetic strategies for producing cyclazine derivatives have been described in the literature [16]-[19]. However, in many cases extended reaction times, elevated temp conditions, and environmentally hazardous organic solvents were used. The synthesis of $\operatorname{cycl}[3,2$, 2]azine in aqueous medium under microwave condition was also reported[20].The present work is a microwave mediated multi-component reactions (MCRs) which constitute an attractive synthetic strategy for rapid and efficient reaction, enhanced reaction rates, cleaner products in a simple way. In continuation of our work for the synthesis of N-heterocycles under microwave condition [21] $\operatorname{cycl}[3,2,2]$ azine derivatives have been synthesised using 2-methyl pyridine, phenacyl bromide derivatives and DMAD in presence of alumina in moderate yields.

\section{Materials and Methods}

Melting points were determined with a Metler melting point apparatus and are uncorrected. All reactions were carried out in a commercially available microwave oven (Samsung M183DN) operating at $300 \mathrm{~W}$. IR spectra were recorded on a Jasco FT/IR -4100 spectrometer using $\mathrm{KBr}$. Mass spectra were recorded with a Waters-3100 spectrometer. ${ }^{1} \mathrm{H}$ NMR spectra were measured in DMSO at room temperature on Brucker Avance III $400 \mathrm{MHz}$ spectrometer. Elemental analyses were conducted on the Elementarvario EL III instrument. All fluorescence measurements were recorded on Jaz Ocean Optics spectrofluorometer. Thin layer chromatography was carried out on aluminium foil coated with silica gel60 F254 (Merck) and column chromatography on silica gel; 70-230 mesh (Merck). UV-visible absorption spectra were studied using Evolution -201 spectrophotometer. All reagents were obtained from commercial sources and used without further purification.

\subsection{Procedure}

$\mathrm{R}=\mathbf{a}) \mathrm{C}_{6} \mathrm{H}_{5}$, b) $\mathrm{C}_{6} \mathrm{H}_{5} \mathrm{NO}_{2}$ c) $\mathrm{C}_{6} \mathrm{H}_{5} \mathrm{Cl}$, d) $\mathrm{C}_{6} \mathrm{H}_{5} \mathrm{OMe}$, e) $\mathrm{C}_{6} \mathrm{H}_{5} \mathrm{Me}$, f) $\mathrm{C}_{6} \mathrm{H}_{5} \mathrm{OH}$, g) $\left.\mathrm{C}_{6} \mathrm{H}_{5} \mathrm{Br}, \mathbf{h}\right) \mathrm{C}_{6} \mathrm{H}_{5} \mathrm{~F}$

To a mixture of $1 \mathrm{mmol}(0.12 \mathrm{~mL}) 2$-picoline (1), $1.2 \mathrm{mmol}$ (240mg) phenacyl bromide $(2)$ and $1.5 \mathrm{mmol}(0.18 \mathrm{~mL})$ $\operatorname{DMAD}(3)$ were added and mixed with $1 \mathrm{mmol}$ (153mg)alumina in an Erlenmeyer flask and is fitted with a bent tube. The other end of the bent tube is connected to a receiver. The reaction mixture was irradiated for 2 minutes at $300 \mathrm{~W}$ with intermittent irradiation for 30 seconds (reaction monitored by TLC). The viscous mass obtained was cooled to room temperature and the products were further purified by column chromatography using n-hexane/EtOAc, $(9: 1 \mathrm{v} / \mathrm{v})$. The products were recrystallized from methanol. 


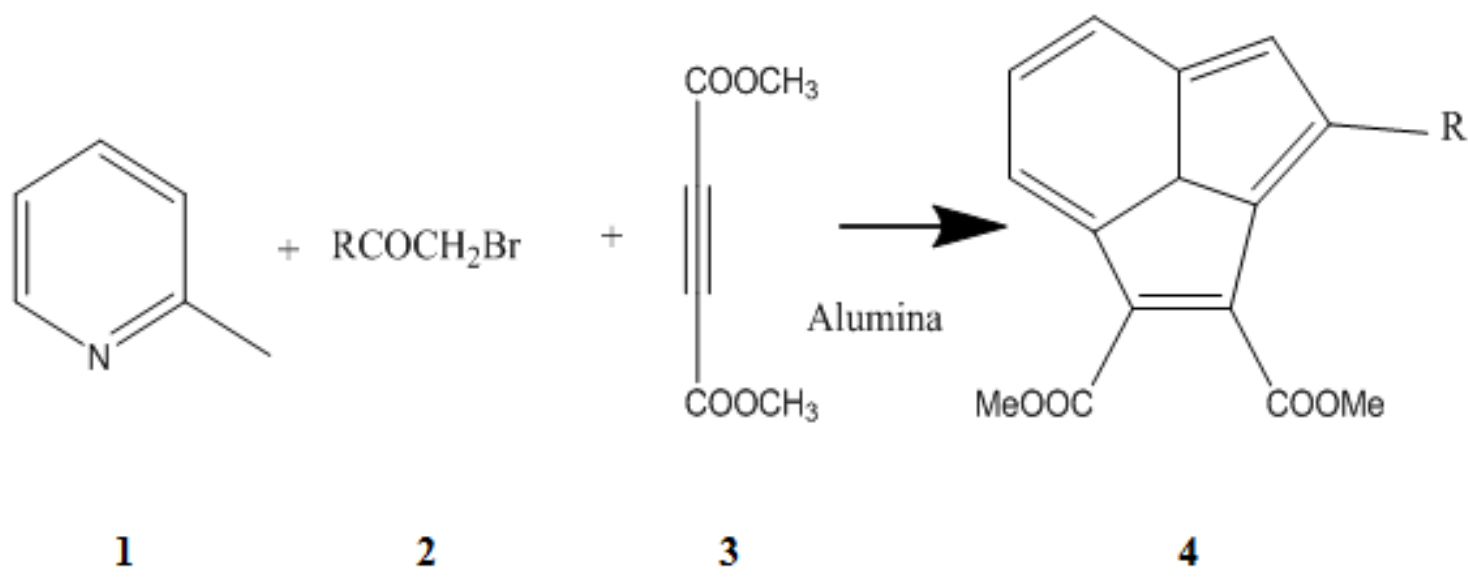

Scheme 1: Microwave synthesis of $\operatorname{cycl}[3,2,2]$ azine derivatives (4a-h)

\section{Results and Discussion}

The cyclazine derivatives (4a-h) synthesised were analysed for IR.UV, ${ }^{1} \mathrm{HNMR},{ }^{13} \mathrm{C}$ NMR and mass spectral techniques. The cycl[3.2.2] azine products (4a-h) were obtained within $2-$ $5 \mathrm{~min}$ in $20-80 \%$ yield. The analytical parameters are represented in Table 1.

Table 1: Analytical parameters of the synthesised compounds

\begin{tabular}{|c|c|c|c|c|}
\hline Compound & $\begin{array}{c}\text { Yield } \\
(\%)\end{array}$ & $\begin{array}{c}\text { M.P } \\
\left({ }^{0} \mathrm{C}\right)\end{array}$ & $\begin{array}{c}M^{+} \text {from } \\
\text { mass } \\
\text { spectra }\end{array}$ & $\begin{array}{c}\text { Molecular } \\
\text { formula }\end{array}$ \\
\hline $4 \mathrm{a}$ & 37 & 140 & 333 & $\mathrm{C}_{20} \mathrm{H}_{15} \mathrm{NO}_{4}$ \\
\hline $4 \mathrm{~b}$ & 78 & 138 & 378 & $\mathrm{C}_{20} \mathrm{H}_{14} \mathrm{~N}_{2} \mathrm{O}_{6}$ \\
\hline $4 \mathrm{c}$ & 46 & 153 & 367.5 & $\mathrm{C}_{20} \mathrm{H}_{14} \mathrm{NO}_{4} \mathrm{Cl}$ \\
\hline $4 \mathrm{~d}$ & 23 & 113 & 363 & $\mathrm{C}_{21} \mathrm{H}_{17} \mathrm{NO}_{5}$ \\
\hline $4 \mathrm{e}$ & 39 & 97 & 347 & $\mathrm{C}_{21} \mathrm{H}_{17} \mathrm{NO}_{4}$ \\
\hline $4 \mathrm{f}$ & 36 & 111 & 349 & $\mathrm{C}_{20} \mathrm{H}_{15} \mathrm{NO}_{5}$ \\
\hline $4 \mathrm{~g}$ & 49 & 123 & 411 & $\mathrm{C}_{20} \mathrm{H}_{14} \mathrm{NO}_{4} \mathrm{Br}$ \\
\hline $4 \mathrm{~h}$ & 42 & 93 & 352 & $\mathrm{C}_{20} \mathrm{H}_{14} \mathrm{NO}_{4} \mathrm{~F}$ \\
\hline
\end{tabular}

It was observed that substrates bearing an electron releasing group at the para position of the aromatic ring (2b) facilitated the enhancement in yields of the products, whereas electron deficient (2d) gave lower yields. The three-component reactions in the absence of solvent were found time consuming when carried out under thermal conditions (1-24 h) in comparison to microwave heating wherein the reactions were very rapid (2-5 $\mathrm{min})$.

\subsection{Fluorescence Activity}

All the products obtained were found to be fluorescent active and further studies were conducted. In presence of metal ions, methoxy $\operatorname{cycl}[3,2,2]$ azine derivative exhibited a quenching effect (Figure 1).

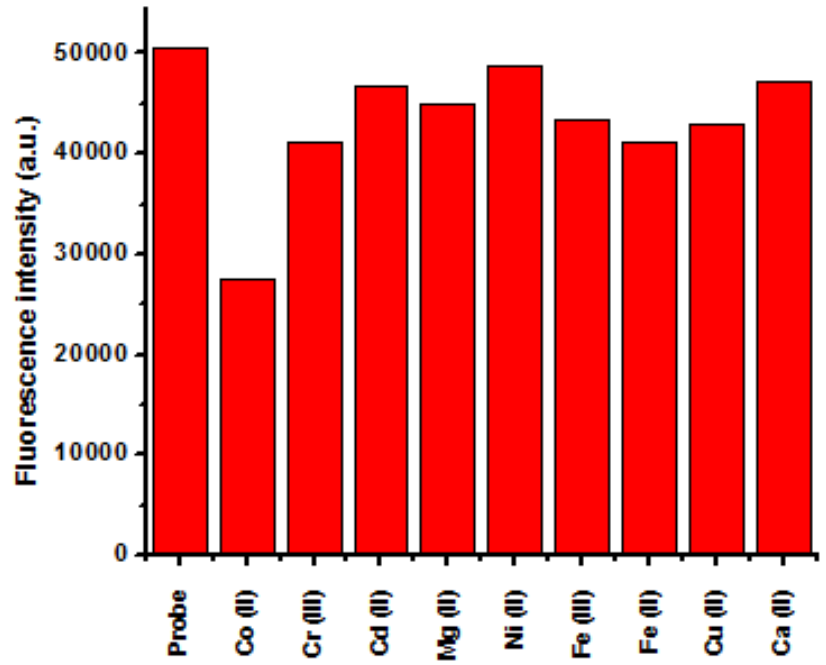

Figure 1: Fluorescence quenching effect of methoxycycl[3, 2,2 ] azine derivative in presence of various metal ions

It was found that methoxy cyclazine derivative can be employed as a fluorophore for developing $\mathrm{Co}^{2+}$ ion sensor [22].

\subsection{Antibacterial Activity}

The activity was measured against 10 pathogenic microorganisms. The antibacterial activity of the compounds were studied against Bacillus cereus, Bacillus pumilus, Bacillus Maculans, Escherichia coli, Klebsiella pneumonia, Proteus vulgaris, Clostridium perfringens, Pseudomonas aeruginosa, Salmonella typhimurium and Staphylococcus aureus. Pure ampicillin was taken as positive control. Antibacterial activity studies of the synthesised derivatives revealed that hydroxy and chloro derivatives showed pronounced antibacterial activity. The bromo and fluoro derivatives showed activity for certain microorganisms only (Table 2). 
International Journal of Science and Research (IJSR)

ISSN (Online): 2319-7064

Index Copernicus Value (2013): 6.14 | Impact Factor (2014): 5.611

Table 2: Antibacterial activity studies of cycl[3, 2, 2]azine derivatives

\begin{tabular}{|l|c|c|c|c|c|c|c|c|}
\hline & \multicolumn{7}{|c|}{ Antibacterial activity of samples 4(a-h) } & $\mathrm{Hr}$ \\
\hline Microorganism & $\mathrm{Me}$ & $\mathrm{OH}$ & $\mathrm{Cl}$ & $\mathrm{OMe}$ & $\mathrm{NO}_{2}$ & $\mathrm{H}$ & $\mathrm{Br}$ & + \\
\hline B. cereus & & ++ & + & & & & ++ & ++ \\
\hline B. pumilus & & ++ & ++ & & & & & + \\
\hline B. maculans & & + & + & & & & + & + \\
\hline Staphylococcus aureus & & ++ & ++ & & + & & & + \\
\hline Salmonella typhimurium & + & ++ & ++ & + & + & + & & ++ \\
\hline E. coli & & + & + & & & & + & \\
\hline Proteus vulgaris & & ++ & + & & & & & + \\
\hline Clostridium perfringens & & + & & & & & + & ++ \\
\hline Klebsiella pneumoniae & & ++ & + & & & & & + \\
\hline Pseudomonas aeruginosa & & + & + & & & & ++ \\
\hline
\end{tabular}

4a)2-Phenyl-4, 5-dicarbmethoxycycl[3.2.2] azine yellow needles;Yield:37\% ;mp $140{ }^{0} \mathrm{C}$; Elemental Analysis for $\mathrm{C}_{20} \mathrm{H}_{15} \mathrm{NO}_{4}$; Calculated: $\mathrm{C}$ 72.68; H 3.75; N 3.6 Found: $\mathrm{C}$ 70.54; H 3.19; N 3.66; IR $\left(\mathrm{KBr} \mathrm{cm} \mathrm{cm}^{-1}\right): 2924,2853,1723$, $1705,1617,1487,1256,1209,1169,1117,1067,760,401$; ${ }^{1} \mathrm{H}$ NMR (DMSO): 7.93-8.42 (4H, m), 7.43-7.52 (5H, m), $5.12(3 \mathrm{H}, \mathrm{s}), 3.57(3 \mathrm{H}, \mathrm{s}) ;{ }^{13} \mathrm{C} \mathrm{NMR}: 190.3,165.6,164.3(2 \mathrm{C})$, $163.8,158.4,145.6,138.4,138.3,130.7,127.2$, 124.6, 124.1, 123.4, 89.6, 66.4, 54.3., 53.4, 52.7, 50.8; Mass $\mathrm{m} / \mathrm{z}: 333$ $[\mathrm{M}+]$.

4b)2-Nitrophenyl-4, 5 dicarbmethoxycycl[3.2.2] azine yellow powder; Yield: $78 \% ; \mathrm{mp} 138^{\circ} \mathrm{C}$;.Elemental Analysis for $\mathrm{C}_{20} \mathrm{H}_{14} \mathrm{~N}_{2} \mathrm{O}_{6}$; Calculated:C57.15;H2.35;N8.9Found:C56.87; $\mathrm{H} 2.49$; N7.13; IR( $\left.\mathrm{KBrcm}^{1}\right): 2924 ， 1733 ， 1698 ， 1610 ， 1545$, 1356, 1176, 1097, 772, 406; ${ }^{1} \mathrm{HNMR}(\mathrm{DMSO}): 7.80(2 \mathrm{H}, \mathrm{d}$, $\mathrm{J}=7.3 \mathrm{~Hz}), 6.32(1 \mathrm{H}), .7 .57(1 \mathrm{H}, \mathrm{m}), 7.11(1 \mathrm{H}, \mathrm{m}), 3.51(3 \mathrm{H}, \mathrm{s})$; ${ }^{13} \mathrm{C}$ NMR :190.1, 166.7(2C), 163.7, 145.9, 138.7, 138.5(2C), $124.4,124.3$ (2C), 123.4, 89.6, 89.1, 66.5, 54.1, 53.1, 52.2, 51.5(2C);Mass $\mathrm{m} / \mathrm{z}: 378[\mathrm{M}+]$.

4c)2-Chlorophenyl-4, 5-dicarbmethoxy-cycl[3.2.2] azine yellow needles; Yield: $46 \%$; mp $153^{\circ} \mathrm{C}$; Elemental AnalysisforC ${ }_{20} \mathrm{H}_{14} \mathrm{NO}_{4} \mathrm{Cl}$;Calculated:C60.21;H4.32;N7.57Fo und:C57.64;H3.33;N7.13;IR(KBrcm1):2924, 1740, 1698, $1610, \quad 1505, \quad 1256, \quad 1176, \quad 1097, \quad 772, \quad 432 ;{ }^{1} \mathrm{H}$, NMR(DMSO):7.67(2H, d, J=7.1Hz), 7.42(1H).7.45(1H, m), 6.34(1H, t), 7.21(1H, m), 3.49(3H, s $){ }^{13} \mathrm{CNMR}: 190.2$, $166.9(2 \mathrm{C}), 162.2,159.0,154.1,145.2,143.6,129.7(2 \mathrm{C})$, $125.5,124.6$ (2C), 123.6, 89.1, 66.5, 54.1, 53.4, 53.2, 52.5(2C); Mass m/z : $367.5[\mathrm{M}+]$.

4d)2-Methoxyphenyl-4, 5-dicarbmethoxy cycl[3.2.2]azine yellow needles; Yield: $23 \%$; mp $113^{\circ} \mathrm{C}$; Elemental Analysis for $\mathrm{C}_{21} \mathrm{H}_{17} \mathrm{NO}_{5}$; Calculated: $\mathrm{C} 62.42 ; \mathrm{H} 3.56 ; \mathrm{N} 6.78$ Found: C 61.36; H 3.09; N 6.54; IR(KBrcm $\left.{ }^{-1}\right): 2924,1738,1698$, 1610, 1505, 1250, 1176, 1097, 772, 436; ${ }^{1} \mathrm{HNMR}(\mathrm{DMSO}): 7.67(2 \mathrm{H}, \quad \mathrm{d}, \quad \mathrm{J}=7.3 \mathrm{~Hz}), \quad 6.42(1 \mathrm{H}$, d) $7.45(1 \mathrm{H}, \mathrm{m}), \quad 6.34(1 \mathrm{H}, \mathrm{t}), \quad 7.21(1 \mathrm{H}, \mathrm{m}), \quad 3.50(3 \mathrm{H}$, s) ${ }^{13}$ CNMR:190.1, 166.3, 163.9(2C), 154.2, 146.0, 145.4, 138.1, 136.1, 135.6, 127.7(2C), 125.9 (2C), 125.4, 87.6, 67.9, 54.5, 53.5(2C); Mass m/z : $363[\mathrm{M}+]$.
4e)2-Methylphenyl-4, 5-dicarbmethoxy-cycl[3.2.2]azine yellow needles; Yield: $39 \%$; mp $97^{\circ} \mathrm{C}$; Elemental Analysis for $\mathrm{C}_{21} \mathrm{H}_{17} \mathrm{NO}_{4}$ Calculated: $\mathrm{C} 56.78 ; \mathrm{H} 4.23 ; \mathrm{N} 4.98$ Found: $\mathrm{C}$ 58.45; H 3.67; N 4.06; IR $\left(\mathrm{KBrcm}^{-1}\right): 2924,2919,1740,1698$, 1610, 1505, 1256, 1176, 1097, 772, $405 ;{ }^{1} \mathrm{HNMR}(\mathrm{DMSO}): 7.68(2 \mathrm{H}, \quad$ d.J $=7.1 \mathrm{~Hz}), .7 .67(1 \mathrm{H}, \quad \mathrm{m})$, 6.32(1H, t), 7.23(1H, m); ${ }^{13} \mathrm{CNMR}: 190.0,166.7,162.9(2 \mathrm{C})$, $150.1,147.0,146.8,136.1,132.2,131.1,124.5(2 \mathrm{C}), 123.6$ (2C), 124.2, 87.6, 68.1, 66.2, 52.5, 20.2 ;Mass m/z : 347 $[\mathrm{M}+]$.

4f)2-Hydroxyphenyl-4, 5- dicarbmethoxycycl[3.2.2]azine yellowneedles;Yield:36\%;mp $111^{\circ} \mathrm{C}$;Elemental Analysis for $\mathrm{C}_{20} \mathrm{H}_{15} \mathrm{NO}_{5}$ Calculated: $\mathrm{C} 60.56$; H 3.24; N 1.89 Found: $\mathrm{C}$ 59.06;H3.83; N2.21;IR $\left(\mathrm{KBrcm}^{-1}\right): 3431 ， 2924 ， 1727 ， 1698$, 1610, 1505, 1256, 1176, 1097, 772, 415; ${ }^{1} \mathrm{HNMR}(\mathrm{DMSO}): 7.64(2 \mathrm{H}, \quad \mathrm{d}, \quad \mathrm{J}=7.2 \mathrm{~Hz})$, 8.52(1H).7.32 $(1 \mathrm{H}, \quad \mathrm{m}), \quad 6.42(1 \mathrm{H}, \quad \mathrm{t}), \quad 7.45(1 \mathrm{H}$, $\mathrm{m}) ;{ }^{13} \mathrm{CNMR}: 190.2,166.4,161.9(2 \mathrm{C}), 154.2,148.0,146.9$, 38.4, 134.1, 130.6, 126.7(2C), 125.2 (2C), 125.8, 88.4, 66.1, $56.2,51.5(2 \mathrm{C}) ;$ Mass m/z : $349[\mathrm{M}+]$.

4g)2-Bromophenyl-4, 5-dicarbmethoxy-cycl[3.2.2]azine yellowneedles; Yield:49\%;mp $123^{\circ} \mathrm{C}$;Elemental Analysis for $\mathrm{C}_{20} \mathrm{H}_{14} \mathrm{NO}_{4} \mathrm{Br}$; Calculated: $\mathrm{C} 71.42 ; \mathrm{H} 4.76$ N 3.43 Found: $\mathrm{C}$ 74.54; H 4.24; N 3.13; IR(KBrcm $\left.{ }^{-1}\right): 2825,1733,1674,1520$, 1578, 1134, 1098, 1021, 807, 716;. ${ }^{1} \mathrm{H}$, NMR(DMSO):7.65(2H, d.J=7.2Hz), 8.22(1H).7.41(1H, m), 6.21(1H, t), 7.17(1H, m); ${ }^{13} \mathrm{CNMR}: 190.1,166.9(2 \mathrm{C}), 154.2$, $146.2, \quad 145.0, \quad 144.6, \quad 143.6,132.7,130.1,127.7(2 \mathrm{C})$, 125.3(2C), 89.7, 66.1, 64.1, 55.2, 54.5(2C); Mass m/z : 411 $[\mathrm{M}+]$.

4h)2-Fluorophenyl-4, 5-dicarbmethoxy-cycl[3.2.2]azine yellow needles; Yield: $42 \%$; mp $93{ }^{\circ} \mathrm{C}$ Elemental Anal. for $\mathrm{C}_{20} \mathrm{H}_{14} \mathrm{NO}_{4} \mathrm{~F}$;Calculated:C 73.22; H 4.02; N 3.13 Found: C 74.14; H 3.74; N 3.87; IR ( $\left.\mathrm{KBr} \mathrm{cm}^{-1}\right): 2951,1737,1545$, 1513, 1478, 1227, 1167, 432; ${ }^{1} \mathrm{HNMR}(\mathrm{DMSO}): 7.66(2 \mathrm{H}$, $\mathrm{J}=7.1 \mathrm{~Hz}), \quad 7.47(1 \mathrm{H}, \quad \mathrm{m}), \quad 6.14(1 \mathrm{H}, \quad \mathrm{t}), \quad 7.05(1 \mathrm{H}$, $\mathrm{m}) ;{ }^{13} \mathrm{CNMR} 190.2,166.6,163.2,156.9(2 \mathrm{C}), 154.8,151.0$, 153.6, 130.6, 124.9, 124.7(2C), 123.3(2C), 122.6, 89.1, 93.2, 54.4, 53.3(2C); Mass m/z: $352[\mathrm{M}+]$ 


\section{International Journal of Science and Research (IJSR) \\ ISSN (Online): 2319-7064}

Index Copernicus Value (2013): 6.14 | Impact Factor (2014): 5.611

\section{Conclusions}

An efficient and convenient method for the synthesis of cyclazines in the absence of solvents and metal catalysts is described. A variety of cyclazine derivatives can be synthesised in moderate yields using this solvent free approach. All the synthesised derivatives were fluorescent and can be applied in sensor development. The biological importances of these classes of compounds are also interesting.

\section{Acknowledgements}

One of the authors (Sheela Gopal M) is thankful to the University Grants Commission (UGC), India, for granting FIP and Department of Applied Chemistry and Department of Biotechnology, CUSAT, Cochin for their technical support.

\section{References}

[1] W. Flitsch, In Comprehensive Heterocyclic Chemistry; A. R. Katritzky, C. W. Rees, Pergamon Press: Oxford, Vol. 4, p 443, 1984.

[2] W. L. Mosby, Heterocyclic systems with bridgehead nitrogen atoms. Part One. (The Chemistry of heterocyclic compounds, a series of monographs) Wiley-Interscience, New York, Vol.1, 1961.

[3] T. Uchida, K. Matsumoto, "Methods for the Construction of the Indolizine Nucleus", Synthesis, 1976(4), 209-236, 1976.

[4] L.D. Bolle, G. Andrei, R. Snoeck, Y. Zhang, A. V. Lommel, M. Otto, A. Bousseau, C. Roy, E. D. Clercq, L. Naesens " Potent, selective and cell-mediated inhibition of human herpesvirus 6 at an early stage of viral replication by the non-nucleoside compound CMV423", Biochemical pharmacology, 67(2), 325-336, 2004.

[5] A. S. Jorgensen, P. Jacobsen, L. B. Christiansen, P. S.Bury, A. Kanstrup, S. M. Thorpe, S. Bain, L. Naerum, K. Wassermann, "Synthesis and pharmacology of a novel pyrrolo[2, 1, 5-cd] indolizine (NNC 45-0095), a high affinity non-steroidal agonist for the estrogen receptor", Bioorganic and Medicinal Chemistry Letters, 10(4), 399-402, 2000.

[6] S. Medda, P. Jaisankar, R. Manna, B. Pal, V. S. Giri, M. K. Basu, "Phospholipid microspheres: a novel delivery mode for targeting antileishmanial agent in experimental leishmaniasis", Journal of Drug Target, 11(2), 123-128, 2003.

[7] P. Wang, Z. Zhang, X. Ma, Y. Huang, X. Liu, P. Tu., T. Tong, "HDTIC-1 and HDTIC-2, two compounds extracted from Astragali Radix, delay replicative senescence of human diploid fibroblasts", Mechanisms of Ageing and Development, 124(10-12), 1025-1034, 2003.

[8] L. L. Gundersen, A. H. Negussie, O.B. Frode Rise, stby, "Antimycobacterial Activity of 1-Substituted Indolizines", Archiv der Pharmazie Pharmaceutical and Medicinal Chemistry, 336((3), 191-195, 2003.

[9] N. Abe, T. Nishiwaki, H. Yamamoto,"Synthesis and regioselective cycloaddition reactions of cyclohept[d] imidazo[1, 2-a]imidazoles $\quad(1, \quad 3 \mathrm{a}, \quad 9$ Triazacyclopent[a]azulenes):formation of 2, 3diazacyclohepta[e, f] cycl[3, 2, 2] azine system", Chemistry Letters, (6), 805-806, 1982.

[10] V. Boekelheide, T. Small, "A Correlation of Some Electrophilic Substitution Reactions of Cycl[3.2.2]azine",
Journal of the American Chemical Society, 83(2), 462-463, 1961.

[11] Y. Abe, H. Kayakiri, S. Satoh, T. Inoue, Y. Sawada, K. Imai, N. Inamura, M. Asano, C. Hatori, A. Katayama, T. Oku, H. Tanaka," A Novel Class of Orally Active NonPeptide Bradykinin $\mathrm{B}_{2}$ Receptor Antagonists. 1. Construction of the Basic Framework", Journal of Medicinal Chemistry, 41(4), 564-578, 1998.

[12] A. Gueiffier, S. Mavel, M. Lhassani, A. Elhakmaoui, R. Snoeck, G. Andrei, O. Chavignon, J. C. Teulade, M. Witvrouw, J. Balzarini, E. D. Clercq, J. P. Chapat, "Synthesis of.Imidazo[1, 2-a $]$ pyridines as Antiviral Agents", Journal of Medicinal Chemistry, 41(25), 51085112, 1998.

[13] J. J. Kaminski, B. Wallmark, C. Briving, B. M. Anderson, "Antiulcer agents. 5. Inhibition of gastric $\mathrm{H}+/ \mathrm{K}(+)$-ATPase by substituted imidazo[1, 2-a]pyridines and related analogues and its implication in modeling the high affinity potassium ion binding site of the gastric proton pump enzyme", Journal of Medicinal Chemistry, 34(2), 533-541, 1991.

[14] R. J. Hodgkiss, R. W. Middleton, J. Parrick, H. K. Rami, P. Wardman. G. D. Wilson," Bioreductive fluorescent markers for hypoxic cells: a study of 2-nitroimidazoles with 1substituents containing fluorescent, bridgehead-nitrogen, bicyclic systems", Journal of Medicinal Chemistry, 35(10), 1920-1926, 1992.

[15] D. A. Lerner, P. M. Horowitz, E. M. Evleth,"Comparative photophysics of indolizine and related heterocyclics", The Journal of Physical Chemistry, 81(1), 12-17, 1977.

[16] A. Galbraith, T. Small, V. Boekelheide, "CommunicationsReaction of Dimethyl Acetylenedicarboxylate with Pyrrocoline", The Journal of Organic Chemistry, 24(4), 582-582, 1959.

[17] A. Galbraith, T. Small, R. A. Barner, V. Boekelheide, "The Formation of Cycl[3.2.2] azine Derivatives via the Reaction of Pyrrocoline with Dimethyl Acetylenedicarboxylate", Journal of the American Chemical Society, 83(2), 453-458, 1961.

[18] J. Godfry, "Communications- Novel Synthesis of a 1, 2, 5, 6-Dibenzocycl[3, 2, 2]azine", The Journal of Organic Chemistry, 24(4), 581-581, 1959.

[19] Y. Tominaga, Y. Shiroshita, T. Kurokawa, H. Gotou, Y. Matsuda, A. Hosomi, "Synthesis of cycl[3.2.2]azine and benzo[ $g] \operatorname{cycl}[3.2 .2]$ azine derivatives by use of the $[2+8]$ cycloaddition reaction of indolizines and dimethyl acetylenedicarboxylate", Journal of Heterocyclic Chemistry, 26(2), 477-487, 1989.

[20] S. Gogoi, M. Dutta, J. Gogoi, R. C. Boruah, "Microwave promoted synthesis of cycl[3.2.2] azines in water via a new three-component reaction", Tetrahedron Letters, 52(7), 813-816, 2011.

[21] M. S. Gopal, I. Anitha, "Aqueous phase microwave synthesis of some bisindolizines", International Journal of Chemical Studies, 2(4), 35-38, 2014.

[22] I. Anitha, M. S. Gopal,"A new fluorescent sensor based on bisindolizine derivative", Journal of Fluorescence, DOI $10.1007 / \mathrm{s} 10895-015-1760-5$ 